\title{
Development of Spectrum Imaging Strategies for Quantitative X-Ray Microanalysis
}

Raynald Gauvin $^{1}$, Pierre Michaud ${ }^{1}$ and Michel L. Trudeau ${ }^{2}$

1.Department of Materials Engineering, McGill University, M. H. Wong Bldg, 3610 University Street, Montreal, Québec, Canada, H3A 2B2.

2.Chimie et Matériaux, Centre de Recherche d'Hydro-Québec, 1800 Boul. Lionel-Boulet, Varennes, Québec, Canada, J3X 1S1.

With the development of the new EDS SDD x-ray detectors, the improvement in the cpu speed of modern computers as well as the increase of the capacity of memory storage, the acquisition of spectrum image consisting of one x-ray spectra per pixel will good acquisition statistics is now a reality. In this context, it is imperative to develop strategies for processing these $3 \mathrm{D}$ data cubes of information in order to perform quantitative x-ray microanalysis and to obtain the determination of regions of similar composition. The determination of the composition from the x-ray intensities is generally performed with the ZAF method in the SEM and the Cliff-Lorimer method in the TEM. However, these two methods work only for homogeneous materials. For heterogeneous materials, multivariate methods based on principal component analysis are now used to determine the different regions or phases in an x-ray data cube acquired with experimental noise. However, these processed spectrums gives regions of similar composition but the next step leading to the composition of the phases is generally omitted because of the limitations of the current quantitative x-ray models.

In this work, Spectrum Imaging strategies are being developed to perform quantitative $x$-ray microanalysis from 3D data cubes with a combination of Monte Carlo simulations and various multivariate analysis techniques based on mixing principal component analysis and Fourier analysis and/or Kolmogorov statistical tests. As an example of this work in progress, a reconstructed spectra of a region of a material made of carbon obtained with Kolmogorov statistics is shown in figure [1]. The total number of counts in these simulated spectra was 100.

This paper with present these various strategies of spectrum imaging with EDS maps obtained with low voltage field emission scanning electron microscopy (Hitachi SU-8000 with Oxford $80 \mathrm{~mm} 2$ SDD EDS detector) and field emission transmission electron microscopy (Hitachi HD-2700 with Cs corrector and Broker SDD EDS detector). 


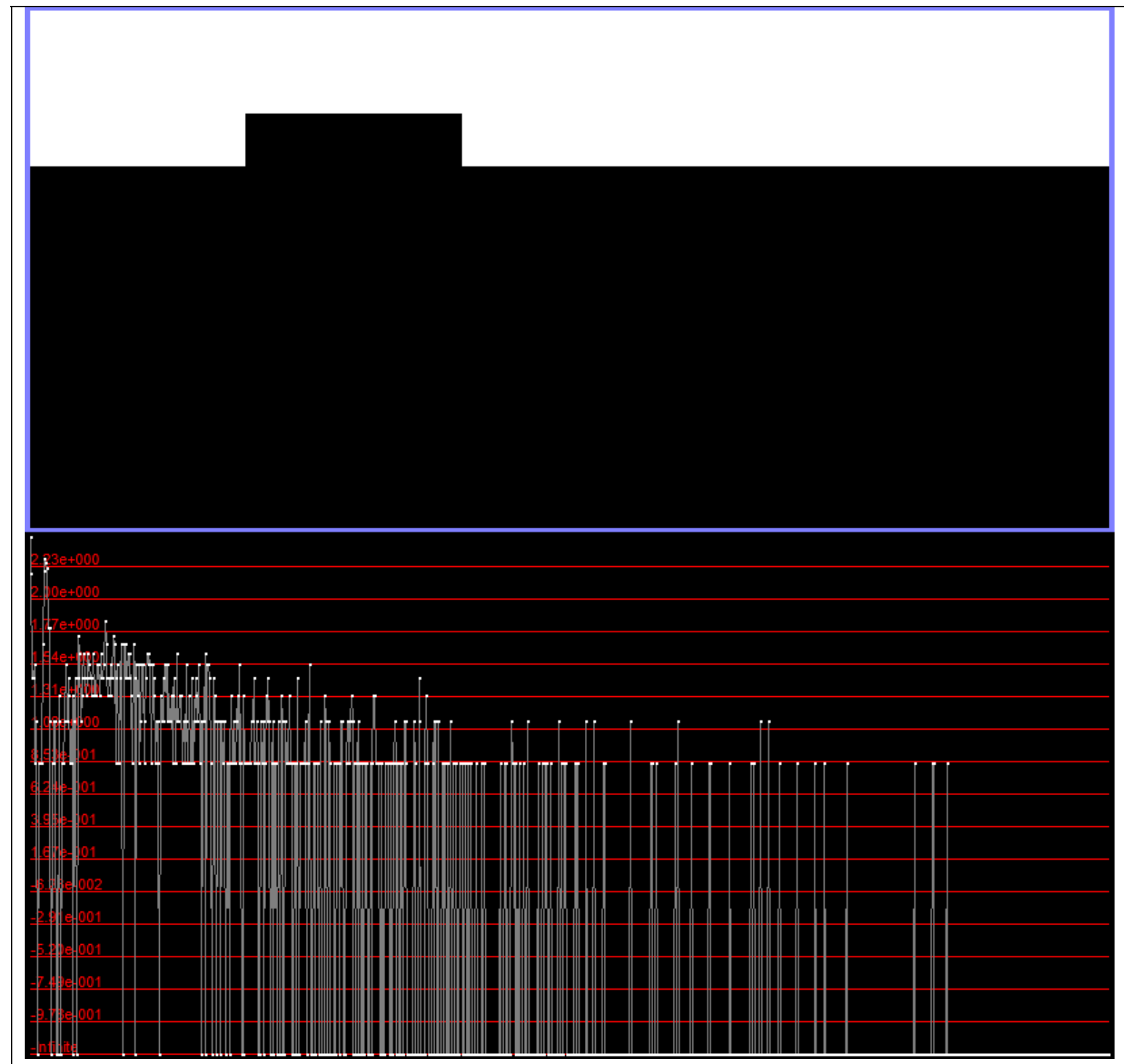

Figure [1] Reconstructed spectra from Kolmogorov statistics of a region rich in carbon. 